\title{
Variational quantum algorithms for nonlinear problems
}

\author{
Michael Lubasch $\odot,{ }^{1}$ Jaewoo Joo, ${ }_{1}^{1}$ Pierre Moinier, ${ }^{2}$ Martin Kiffner $\odot,{ }^{3,1}$ and Dieter Jaksch ${ }^{1,3}$ \\ ${ }^{1}$ Clarendon Laboratory, University of Oxford, Parks Road, Oxford OX1 3PU, United Kingdom \\ ${ }^{2}$ BAE Systems, Computational Engineering, Buckingham House, FPC 267, PO Box 5, Filton, Bristol BS34 7QW, United Kingdom \\ ${ }^{3}$ Centre for Quantum Technologies, National University of Singapore, 3 Science Drive 2, Singapore 117543
}

(Received 29 July 2019; revised manuscript received 11 December 2019; published 6 January 2020)

\begin{abstract}
We show that nonlinear problems including nonlinear partial differential equations can be efficiently solved by variational quantum computing. We achieve this by utilizing multiple copies of variational quantum states to treat nonlinearities efficiently and by introducing tensor networks as a programming paradigm. The key concepts of the algorithm are demonstrated for the nonlinear Schrödinger equation as a canonical example. We numerically show that the variational quantum ansatz can be exponentially more efficient than matrix product states and present experimental proof-of-principle results obtained on an IBM Q device.
\end{abstract}

DOI: 10.1103/PhysRevA.101.010301

Nonlinear problems are ubiquitous in all fields of science and engineering and often appear in the form of nonlinear partial differential equations (PDEs). Standard numerical approaches seek solutions to PDEs on discrete grids. However, many problems of interest require extremely large grid sizes to achieve accurate results, in particular in the presence of unstable or chaotic behavior that is typical for nonlinear problems [1-3]. Examples include large-scale simulations for reliable weather forecasts [4-6] and computational fluid dynamics [7-9].

Quantum computers promise to solve problems that are intractable on conventional, i.e., standard classical, computers through their quantum-enhanced capabilities. In the context of PDEs, it has been realized that quantum computers can solve the Schrödinger equation faster than conventional computers [10-12] and these ideas have been generalized recently to other linear PDEs [13-18]. However, nonlinear problems are intrinsically difficult to solve on a quantum computer due to the linear nature of the underlying framework of quantum mechanics.

Recently, the concept of variational quantum computing (VQC) has attracted considerable interest [19-32] for solving optimization problems. Variational quantum computing is a quantum-classical hybrid approach where the evaluation of the cost function $\mathcal{C}(\boldsymbol{\lambda})$ is delegated to a quantum computer, while the optimization of variational parameters $\lambda$ is performed on a conventional classical computer. The concept of VQC has been applied, e.g., to simulating the dynamics of strongly correlated electrons through nonequilibrium dynamical mean field theory [22,23,33,34], and quantum chemistry calculations were successfully carried out on existing noisy superconducting [21,25,26] and ion quantum computers [28].

We extend and adapt the concept of VQC to solving nonlinear problems efficiently on a quantum computer by virtue of two key concepts. First, we introduce a quantum nonlinear processing unit (QNPU) that efficiently calculates nonlinear functions of the form $F=f^{(1)^{*}} \prod_{j=1}^{r}\left(O_{j} f^{(j)}\right)$ for VQC. Measuring the ancilla qubit connected to the QNPU as shown in Fig. 1(a) directly yields the sum of all function values $\sum_{k} \operatorname{Re}\left\{F_{k}\right\}$, where $\operatorname{Re}\{\cdot\}$ denotes the real part. The functions $f^{(j)}$ are encoded in variational $n$-qubit states $\left|\psi(\boldsymbol{\lambda})_{j}\right\rangle=\hat{U}_{j}(\boldsymbol{\lambda})|\mathbf{0}\rangle$ created by networks of the form shown in Fig. 1(b). The same function $f^{(i)}=f^{(j)}$ may appear multiple times by choosing $\hat{U}_{i}(\lambda)=\hat{U}_{j}(\lambda)$. Second, we use tensor networks as a programming paradigm for QNPUs to create optimized circuits that efficiently calculate linear operators $O_{j}$ acting on functions $f^{(j)}$. In this way all quantum resources of nonlinear VQC scale polynomially with the number of qubits, which represents an exponential reduction compared with some conventional algorithms.

The variational states $\left|\psi(\lambda)_{j}\right\rangle$ represent $N=2^{n}$ values of the functions $f^{(j)}$ which form a trial solution to the problem of interest. The cost function $\mathcal{C}(\lambda)$ for nonlinear VQC is built up from outputs of different QNPUs that are then processed classically to iteratively determine the optimal set $\lambda$. Large grid sizes that are intractable on a conventional computer require only $n \gtrsim 20$ qubits, which is within the reach of noisy intermediate-scale quantum (NISQ) devices. In addition, the scheme is applicable to other types of nonlinear problems that can be solved via the minimization of a cost function $\mathcal{C}(\lambda)$ [36].

We demonstrate the concept and performance of nonlinear VQC by emulating it classically for the canonical example of the time-independent one-dimensional nonlinear Schrödinger equation

$$
\left[-\frac{1}{2} \frac{d^{2}}{d x^{2}}+V(x)+g|f(x)|^{2}\right] f(x)=E f(x),
$$

where $V$ is an external potential and $g$ denotes the strength of the nonlinearity. We also implement nonlinear VQC for Eq. (1) on IBM quantum computers to establish its feasibility on current NISQ devices. Proof-of-principle results are shown in Figs. 1(c) and 1(d), demonstrating excellent agreement with numerically exact solutions. The nonlinear Schrödinger equation and its generalization to higher dimensions describes various physical phenomena ranging from Bose-Einstein condensation to light propagation in nonlinear media [37-42]. In particular, below we consider Eq. (1) with quasiperiodic 

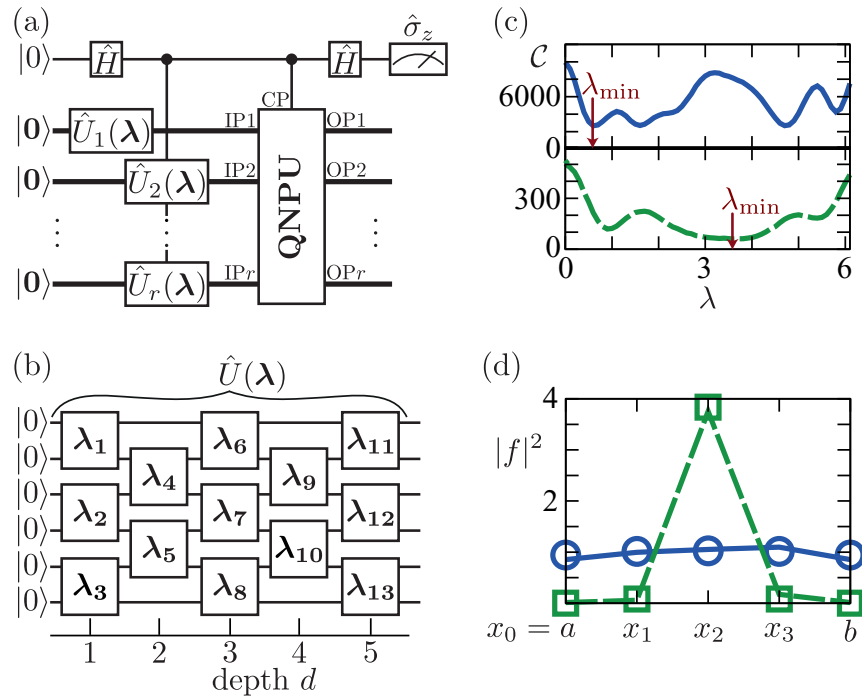

FIG. 1. (a) Quantum network for summing a nonlinear function of the form $F=f^{(1)^{*}} \prod_{j=1}^{r}\left(O_{j} f^{(j)}\right)$. The ancilla qubit on the top line undergoes Hadamard gates $\hat{H}$ and controls operations of the rest of the network via the control port $(\mathrm{CP})$ and is measured in the computational basis. Starting from product states $|\mathbf{0}\rangle$ of $n$ qubits shown as thick lines, variational states $\left|\psi(\boldsymbol{\lambda})_{j}\right\rangle=\hat{U}_{j}(\boldsymbol{\lambda})|\mathbf{0}\rangle$ representing the functions $f^{(j)}$ are created and fed to the QNPU through input ports (IPs). The QNPU contains problem-specific quantum networks defining the linear operators $O_{j}$ and has the output ports (OPs). (b) Network $\hat{U}(\lambda)$ of depth $d=5$ with $n=6$. The values $\lambda=\left\{\lambda_{1}, \lambda_{2}, \ldots\right\}$ determine the form of the two-qubit gates. (c) Cost function $\mathcal{C}(\lambda)$ for the nonlinear Schrödinger equation (1) for a harmonic potential $V$, a single variational parameter $\lambda$, and $g=10^{4}$ (solid line) and $g=10$ (dashed line). The arrows indicate optimal values $\lambda=\lambda_{\text {min }}$. (d) Solutions $|f(x)|^{2}$ for $\lambda=\lambda_{\text {min }}$ with $N=4$ grid points and periodic boundary conditions $f(b)=f(a)$ for $g=10^{4}$ (solid line) and $g=10$ (dashed line). The circles and squares are numerically exact results. Experimental data in (c) and (d) were obtained, from a modified circuit, on IBM Q devices (see [35] for details).

potentials $V$ that are in the focus of current cold-atom experiments [43] and that make Eq. (1) challenging to solve numerically. The methods used for this equation here are straightforwardly modified to handle other nonlinear terms and time-dependent problems as illustrated in [35] for the Burgers equation appearing in fluid dynamics.

The ground state of Eq. (1) can be found by minimizing the cost function

$$
\mathcal{C}=\langle\langle K\rangle\rangle_{c}+\langle\langle P\rangle\rangle_{c}+\langle\langle I\rangle\rangle_{c},
$$

where $\langle\langle K\rangle\rangle_{c},\langle\langle P\rangle\rangle_{c}$, and $\langle\langle I\rangle\rangle_{c}$ are the mean kinetic, potential, and interaction energies, respectively. In Eq. (2) the $\langle\langle\cdot\rangle\rangle_{c}$ denote averages with respect to a single real-valued function $f^{(1)} \equiv f$ on the interval $[a, b]$ satisfying the normalization condition $\int_{a}^{b}|f(x)|^{2} d x=1$.

In line with standard numerical approaches [44-46], we apply the finite-difference method (FDM) to Eq. (1) and discretize the interval $[a, b]$ into $N$ equidistant grid points $x_{k}=a+h_{N} k$, where $h_{N}=\ell / N$ is the grid spacing, $\ell=b-a$ is the length of the interval, and $k \in\{0, \ldots, N-1\}$. Each grid point is associated with a variational parameter $f_{k}$ that approximates the continuous solution $f\left(x_{k}\right)$ at $x_{k}$. Furthermore, we impose periodic boundary conditions, i.e., $f_{N}=f_{0}$, and the normalization condition imposed on the continuous functions $f$ translates to

$$
1=h_{N} \sum_{k=0}^{N-1}\left|f_{k}\right|^{2}=\sum_{k=0}^{N-1}\left|\psi_{k}\right|^{2},
$$

where $\psi_{k}=\sqrt{h_{N}} f_{k}$. Note that the condition on the set of parameters $\left\{\psi_{k}\right\}$ is independent of the grid spacing, and in the following we consider optimizing the cost function with respect to them.

All averages $\left\langle\langle\cdot\rangle_{c}\right.$ in Eq. (2) can be approximated by corresponding expressions of the discrete problem $\langle\langle\cdot\rangle\rangle$. We find $[44-46]\langle\langle\cdot\rangle\rangle_{c}=\langle\langle\cdot\rangle\rangle+\mathcal{E}_{\text {grid }}$, where $\mathcal{E}_{\text {grid }} \propto 1 / N^{2}$ is the error associated with the trapezoidal rule when transforming integrals into sums and

$$
\begin{aligned}
\langle\langle K\rangle\rangle & =-\frac{1}{2} \frac{1}{h_{N}^{2}} \sum_{k=0}^{N-1} \psi_{k}^{*}\left(\psi_{k+1}-2 \psi_{k}+\psi_{k-1}\right), \\
\langle\langle P\rangle\rangle & =\sum_{k=0}^{N-1}\left[\psi_{k}^{*} V\left(x_{k}\right) \psi_{k}\right], \\
\langle\langle I\rangle\rangle & =\frac{1}{2} \frac{g}{h_{N}} \sum_{k=0}^{N-1}\left|\psi_{k}\right|^{4} .
\end{aligned}
$$

Note that $\langle\langle K\rangle\rangle$ in Eq. (4a) uses an FDM representation of the second-order derivative in Eq. (1).

To evaluate the terms in Eq. (4) on a quantum computer we consider quantum registers with $n$ qubits and basis states $|\boldsymbol{q}\rangle=\left|q_{1} \cdots q_{n}\right\rangle=\left|q_{1}\right\rangle \otimes \cdots \otimes\left|q_{n}\right\rangle$, where $q_{j} \in\{0,1\}$ denotes the computational states of qubit $j$. Regarding the sequence $q_{1} \ldots q_{n}=$ binary $(k)$ as the binary representation of the integer $k=\sum_{j=1}^{n} q_{j} 2^{n-j}$, we encode all $N=2^{n}$ amplitudes $\psi_{k}$ in the normalized state

$$
|\psi\rangle=\sum_{k=0}^{N-1} \psi_{k}|\operatorname{binary}(k)\rangle .
$$

We prepare the quantum register in a variational state $|\psi(\lambda)\rangle$ via the quantum circuit $\hat{U}(\lambda)$ of depth $d$ shown in Fig. 1(b). We consider depths $d \propto \operatorname{poly}(n)$ such that the quantum ansatz requires exponentially fewer parameters $\mathcal{N}$ than standard classical schemes with $N$ parameters. Note that the number of variational parameters scales like $\mathcal{N} \propto n d$ for our quantum ansatz [35]. The power of this ansatz is rooted in the fact that it encompasses all matrix product states (MPSs) [47-50] with bond dimension $\chi \sim \operatorname{poly}(n)$ [35]. Since polynomials and Fourier series [51,52] can be efficiently represented by MPSs, the quantum ansatz simultaneously contains universal basis functions that are capable of approximating a large class of solutions to nonlinear problems efficiently. Furthermore, we show below that the quantum ansatz is capable of storing solutions with exponentially fewer resources than the classically optimized MPS ansatz. Note that the number of variational parameters scales like $\mathcal{N} \propto n \chi^{2}$ for MPSs of bond dimension $\chi[35]$.

Figure 2(a) demonstrates the basic working principle of the QNPU for the nonlinear term $\langle\langle I\rangle\rangle$. The effect of the 

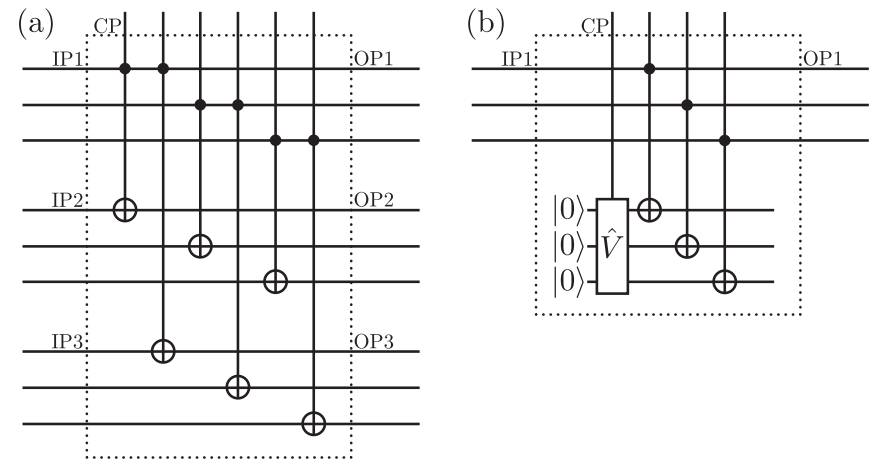

FIG. 2. (a) QNPU circuit calculating the nonlinear term $|\psi|^{4}$. The networks are shown for $n=3$ and all input ports are fed the same variational quantum states created by $\hat{U}(\lambda)$. IP2 is fed $\hat{U}(\lambda)$ and IP3 is fed $\hat{U}^{*}(\lambda)$. (b) QNPU circuit for working out the potential energy term $\tilde{V}|\psi|^{2}$.

controlled-NOT operations between pairs of qubits is to provide a pointwise multiplication with the ancilla thus measuring $\sum_{k}\left|\psi_{k}\right|^{4}$. In Fig. 2(b) we show the circuit for measuring $\langle\langle P\rangle\rangle$. The unitary $\hat{V} \hat{=} O_{1}$ encodes function values of the external potential $V$. A copy of $\psi$ is effectively multiplied pointwise with the external potential by controlled-NOT gates to give $\sum_{k} \tilde{V}_{k}\left|\psi_{k}\right|^{2}$. Similarly, multiplying $\psi$ with their shifted versions using adder circuits (see [35] for details) allows evaluating the kinetic energy term.

The measured expectation value of the ancilla qubit is directly related to the desired quantities as $\langle\langle I\rangle\rangle=g\left\langle\hat{\sigma}_{z}\right\rangle_{\text {anc }}^{I} / 2 h_{N}$ for the nonlinear term, $\langle\langle P\rangle\rangle=\alpha\left\langle\hat{\sigma}_{z}\right\rangle_{\text {anc }}^{P}$ for the potential energy, and $\langle\langle K\rangle\rangle=\left(1-\left\langle\hat{\sigma}_{z}\right\rangle_{\text {anc }}^{K}\right) / h_{N}^{2}$ for the kinetic energy $[53,54]$. Furthermore, derivatives of the cost function, as required by some minimization algorithms [36,55,56], can be evaluated by combining the ideas presented here with the quantum circuits discussed in [24,30,57,58].

The unitary network $\hat{V}$ represents scaled function values $\tilde{V}_{k}$ of the external potential where $\sum_{k=0}^{N-1}\left|\tilde{V}_{k}\right|^{2}=1$, and $\alpha>0$ is a scaling parameter such that $V_{k}=\alpha \tilde{V}_{k}$. Efficient quantum circuits $\hat{V}$ for measuring $\langle\langle P\rangle\rangle$ can be systematically obtained by establishing tensor networks as a programming paradigm. To this end we expand the external potential in polynomials or Fourier series $\tilde{V}(x) \approx \sum_{j}^{J} c_{j} b_{j}(x)$, where $b_{j}(x)$ are basis functions and $c_{j}$ are expansion coefficients [35]. In the case of Fourier series of order $J$, the approximate potential is represented by an MPS of bond dimension $\chi=J$ [51,52]. Next we write the MPSs in terms of $n-\lceil\log \chi\rceil$ unitaries [59-61], where $\lceil\cdot\rceil$ is the ceiling function. Each of these unitaries acts on $2 \chi$ qubits and can be decomposed in terms of elementary two-qubit gates [35,62-65]. An upper bound for the depth of the resulting quantum circuit is $d_{>} \leqslant 9 n\left[\frac{23}{48}(2 \chi)^{2}+\frac{4}{3}\right]$ [64]. The depth thus scales polynomially with the number of qubits $n$ and with $\chi$, and many problems of interest show an even more advantageous scaling. For example, in the following we consider the potential

$$
V(x)=s_{1} \sin \left(\kappa_{1} x\right)+s_{2} \sin \left(\kappa_{2} x\right)
$$

and set $\kappa_{2}=2 \kappa_{1} /(1+\sqrt{5})$. This potential realizes an incommensurate bichromatic lattice where the ratio $s_{1} / s_{2}$ determines the amount of disorder in the lattice [66]. The trap potential
$V(x)$ in Eq. (6) is exactly represented by an MPS of bond dimension $\chi=4$. The depth of the corresponding quantum circuit $d=5(n-2)+1 \ll d_{>}$is much smaller than the upper bound [35].

Next we analyze the Monte Carlo sampling error [44] associated with the measurement of the ancilla qubit. We denote the absolute sampling error associated with quantity $X$ by $\mathcal{E}_{\mathrm{MC}}^{X}$ and the corresponding relative error is [35]

$$
\begin{aligned}
\epsilon_{\mathrm{MC}}^{P} & =\frac{\mathcal{E}_{\mathrm{MC}}^{P}}{\langle\langle P\rangle\rangle}=C_{P} \frac{1}{\sqrt{M}}, \\
\epsilon_{\mathrm{MC}}^{K} & =\frac{\mathcal{E}_{\mathrm{MC}}^{K}}{\langle\langle K\rangle\rangle} \approx C_{K} \frac{N}{N_{\min }} \frac{1}{\sqrt{M}}, \\
\epsilon_{\mathrm{MC}}^{I} & =\frac{\mathcal{E}_{\mathrm{MC}}^{I}}{\langle\langle I\rangle\rangle} \approx C_{I} \frac{N}{N_{\min }} \frac{1}{\sqrt{M}} .
\end{aligned}
$$

In these equations, we assume $N \geqslant N_{\min }$ and $N_{\min }=$ $\ell / \ell_{\min }$ is the minimal number of grid points for resolving the smallest length scale $\ell_{\min }$ of the problem. The parameters $C_{X}$ in Eqs. (7) are of the order of unity [35] and all sampling errors decrease with the number of samples $M$ as $1 / \sqrt{M}$. While the relative error associated with the potential term in Eq. (7a) is independent of the number of grid points, $\epsilon_{\mathrm{MC}}^{K}$ and $\epsilon_{\mathrm{MC}}^{I}$ increase linearly with $N / N_{\min }$. It follows that increasing the grid size requires larger values of $M$ in order to keep the sampling error small. However, the grid error scales like $\mathcal{E}_{\text {grid }} \propto 1 / N^{2}=2^{-2 n}$ for $N \geqslant N_{\text {min }}$ [35]. We thus conclude that only moderate ratios $N / N_{\min }>1$ and therefore relatively small values of $M$ are needed in order to achieve accurate solutions with small grid errors.

The quantum ansatz in Fig. 1(b) is inspired from tensor network theory and can be regarded, for example, as the Trotter decomposition of the time $t$ evolution operator $\exp [-i H(t) t / \hbar]$ of a time-dependent spin Hamiltonian $H(t)$ with arbitrary short-range interactions acting on the initial state $|\mathbf{0}\rangle$ [67]. Similarly to the coupled cluster ansatz in quantum chemistry VQC calculations [19], there is currently no known efficient classical ansatz for this state [68,69]. From the VQC perspective, our quantum ansatz in Fig. 1(b) is composed of generic two-qubit gates that in an experiment are accurately approximated by short sequences of gates if a sufficiently tunable or universal gate set is experimentally available [70]. We envisage that this quantum ansatz is more efficient than methods based on an MPS ansatz on a classical computer like the multigrid renormalization (MGR) method in [56]. This MGR method is the most efficient and accurate classical algorithm known to us for the problem considered here, for which it can already be exponentially faster than standard classical algorithms. A comparison with this powerful classical method, which is based on variational classical MPSs, will allow us to validate the superior variational power of the quantum ansatz in Fig. 1(b) on a quantum computer. Note that the difficulty of solving a classical optimization problem with many variables does not go away by using the quantum ansatz, as the actual optimization is classical and there is no quantum advantage there. The quantum advantage stems solely from the faster evaluation of the cost function for our quantum ansatz in Fig. 1(b) on a quantum computer. 

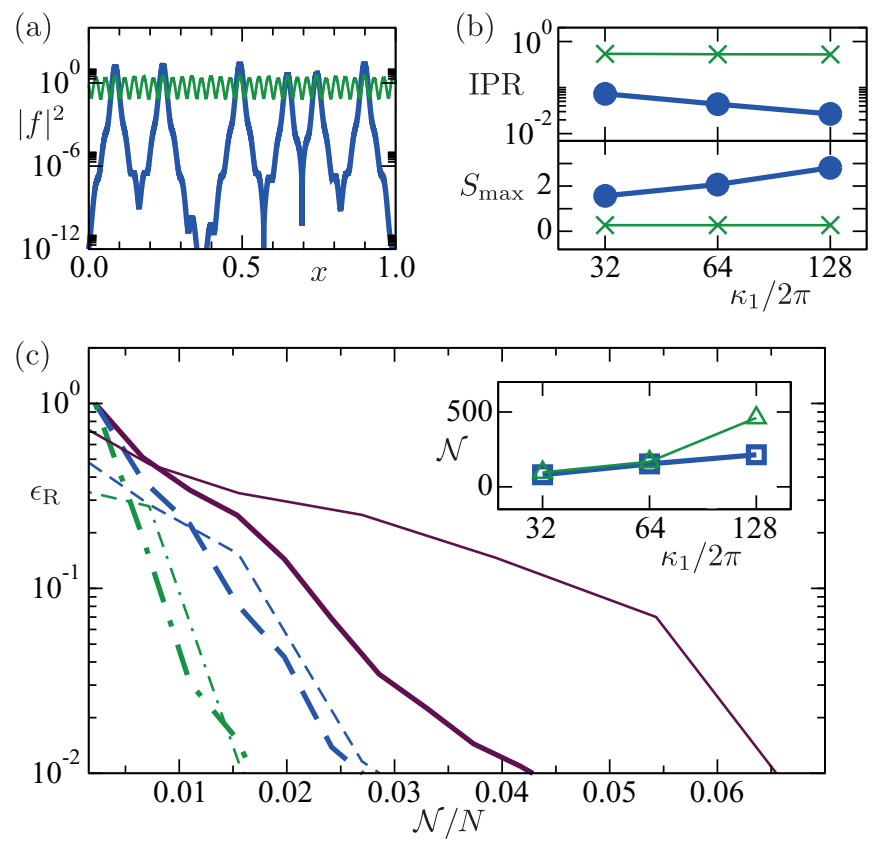

FIG. 3. (a) Numerically exact solution $|f(x)|^{2}$ of Eq. (1) on a logarithmic scale, $V(x)$ in Eq. (6) with $s_{1}=2 \times 10^{4}$ and $\kappa_{1}=2 \pi \times 32$. The green thin line (blue thick line) is for $s_{1} / s_{2}=200\left(s_{1} / s_{2}=2\right)$. (b) A log-log plot of the IPR (top panel) and lin-log plot of $S_{\max }$ of the exact solution $\left|\psi^{\text {exact }}\right\rangle$ (bottom panel) as a function of $\kappa_{1}$. Green crosses (blue circles) correspond to $s_{1} / s_{2}=200\left(s_{1} / s_{2}=2\right)$. (c) Representation error $\epsilon_{\mathrm{R}}$ of the exact solution for the quantum ansatz (thick lines) and the MPS ansatz (thin lines) as a function of $\mathcal{N} / N$. All curves are for $s_{1} / s_{2}=2$ and correspond to $s_{1}=2 \times 10^{4}$ and $\kappa_{1}=2 \pi \times 32$ (green dash-dotted line), $s_{1}=8 \times 10^{4}$ and $\kappa_{1}=$ $2 \pi \times 64$ (blue dashed line), and $s_{1}=3.2 \times 10^{5}$ and $\kappa_{1}=2 \pi \times 128$ (purple solid line). The inset shows $\mathcal{N}$ as a function of $\kappa_{1}$ for $\epsilon_{\mathrm{R}}=0.05$ and $s_{1} / s_{2}=2$. Blue squares (green triangles) correspond to the quantum ansatz (MPS ansatz). All curves in (a)-(c) are for $N=2^{13}=8192$ grid points and $g=50$.

To provide numerical evidence for this we first obtain the numerically exact solution of Eq. (1) on the interval $[0,1]$ via the MGR algorithm [56] and by allowing for the maximal bond dimension $\chi$ of the MPS ansatz [35]. In this case the numerically exact solution is described by $N=2^{n}$ parameters like in other conventional algorithms. The results are shown in Fig. 3(a) for two different values of $s_{1} / s_{2}$. In the weakly disordered regime $s_{1} / s_{2} \gg 1,|f(x)|^{2}$ varies on the length scale set by $1 / \kappa_{1}$. On the contrary, the strongly disordered regime $s_{1} / s_{2} \approx 1$ is characterized by strongly localized solutions in space. The localization of the wave function can be quantified using the inverse participation ratio (IPR) [71] IPR $=\left(N \sum_{k=0}^{N-1}\left|\psi_{k}\right|^{4}\right)^{-1}$. We show the IPR in Fig. 3(b) as a function of $\kappa_{1}$ (top panel) and find that it stays constant for $s_{1} / s_{2} \gg 1$. On the other hand, the IPR decreases according to a power law with $\kappa_{1}$ for $s_{1} / s_{2} \approx 1$, showing that the localized character of the wave function increases dramatically with $\kappa_{1}$.

Next we encode the function values of the numerically exact solution in the state $\left|\psi^{\text {exact }}\right\rangle$ via Eq. (5) and calculate the maximum bipartite entanglement entropy $S_{\max }$ of all possible bipartitions of the $n$-qubit wave function $\left|\psi^{\text {exact }}\right\rangle$. The quantity
$S_{\max }$ is a measure of the entanglement of $\left|\psi^{\text {exact }}\right\rangle$ and is shown in the bottom panel of Fig. 3(b). The value of $S_{\max }$ is small and stays constant with $\kappa_{1}$ for $s_{1} / s_{2} \gg 1$ in the weakly disordered regime. Contrary to this, for $s_{1} / s_{2} \approx 1$ in the strongly disordered regime we observe that $S_{\max } \propto \log \left(\kappa_{1}\right)$.

The entanglement measure $S_{\max }$ provides a useful necessary criterion for efficient MPS approximations [72]. A MPS of bond dimension $\chi$ can at most contain an amount of entanglement $S_{\max }\left[\right.$ MPS] $\leqslant \log _{2}(\chi)$ [47,50]. For MPSs to be efficient, we require that $\chi$ scales at most polynomially with $n$, i.e., $\chi=\operatorname{poly}(n)$, so that $S_{\max }[\operatorname{MPS}] \leqslant \log [\operatorname{poly}(n)]$ and therefore MPS can only capture small amounts of entanglement efficiently. The small values of $S_{\max }$ for $s_{1} / s_{2} \gg 1$ suggest that MPSs work well in the weakly disordered regime and indeed we have confirmed numerically that this is true. Therefore, in the following we focus on the strongly disordered regime $s_{1} / s_{2} \approx 1$. In this regime MPS cannot be an efficient approximation for large values of $\kappa_{1}$ : The total number of variational parameters of MPSs $\mathcal{N}$ [MPS] depends quadratically on $\chi$ [35], i.e., $\mathcal{N}[\mathrm{MPS}] \propto \chi^{2}$, and $\chi$ needs to grow polynomially with $\kappa_{1}$, i.e., $\chi \propto \operatorname{poly}\left(\kappa_{1}\right)$, to satisfy the observed entanglement scaling, such that $\mathcal{N}[\mathrm{MPS}] \propto \operatorname{poly}\left(\kappa_{1}\right)$. Our quantum ansatz (QA) of Fig. 1(b) can capture much larger amounts of entanglement $S_{\max }[\mathrm{QA}] \lesssim d$ efficiently [69] and therefore this ansatz can be an efficient approximation for large values of $\kappa_{1}$ : Because the total number of variational parameters $\mathcal{N}$ depends linearly on $d$ for the quantum ansatz [35], i.e., $\mathcal{N}[\mathrm{QA}] \propto d$, and $d$ just needs to grow logarithmically, i.e., $d \propto \log \left(\kappa_{1}\right)$, for the observed entanglement requirements, we conclude that $\mathcal{N}[\mathrm{QA}] \propto \log \left(\kappa_{1}\right)$. These entanglement considerations show that the quantum ansatz has the potential to be exponentially more efficient than MPSs in the strongly disordered regime for increasing values of $\kappa_{1}$.

To quantitatively analyze and demonstrate the efficiency of the quantum ansatz in this regime, we obtain the set of parameters $\lambda$ that maximize the fidelity $\mathcal{F}=\left|\left\langle\psi^{\text {exact }} \mid \psi(\lambda)\right\rangle\right|$ for different depths $d$ [35]. The infidelity $\epsilon_{\mathrm{R}}=1-\mathcal{F}$ is thus a measure of the error when approximating the exact solution by this ansatz, and in the following we refer to $\epsilon_{R}$ as the representation error. As shown in Fig. 3(c), the representation error decreases exponentially as a function of $\mathcal{N}$ for all values of $\kappa_{1}$ and therefore we obtain accurate solutions for $\mathcal{N} / N \ll 1$. Even for the largest value of $\kappa_{1}=2 \pi \times 128$ and $\epsilon_{\mathrm{R}} \approx 10^{-2}$, we find $\mathcal{N} / N \approx 0.04$, so we require only $4 \%$ of the full number of parameters needed in conventional algorithms. Most importantly, the inset of Fig. 3(c) shows that, to obtain a fixed representation error of $\epsilon_{\mathrm{R}}=0.05$, the number of parameters of our quantum ansatz needs to grow as $\mathcal{N}[\mathrm{QA}] \propto$ $\log \left(\kappa_{1}\right)$. This numerical analysis therefore confirms our expectation, from the entanglement arguments in the preceding paragraph, that the quantum ansatz efficiently approximates solutions in the strongly disordered regime even for large values of $\kappa_{1}$. This is possible because the quantum ansatz captures the required entanglement $S_{\max } \propto \log \left(\kappa_{1}\right)$ by means of just the small number of parameters $\mathcal{N}[\mathrm{QA}] \propto \log \left(\kappa_{1}\right)$. The entanglement capabilities of MPSs imply that $\mathcal{N}[$ MPS $] \propto$ $\operatorname{poly}\left(\kappa_{1}\right)$ has to be fulfilled. Therefore, we conclude that our quantum ansatz is exponentially more efficient than MPSs in the strongly disordered regime for growing values of $\kappa_{1}$. This 
key finding shows that nonlinear VQC can be exponentially more efficient than optimized classical variational schemes that are based on the MPS ansatz.

We now propose a calculation that illustrates the exponential advantage of our quantum ansatz on a quantum computer particularly clearly. We consider the ground-state problem of Eq. (1) with the quasirandom external potential of Eq. (6) on the interval $[0,1]$ discretized by $N=2^{n}$ equidistant grid points and use the FDM from above that leads to Eqs. (4a)-(4c). We choose $\kappa_{1}=2 \pi \times 2^{n / 2}$ and assume that $n=4,6,8, \ldots$ qubits store the variational quantum ansatz. The smallest wavelength present in our external potential of Eq. (6) is determined by $\kappa_{1}$ and reads $2 \pi / \kappa_{1}=2^{-n / 2}$. The interval $[0,1]$ accommodates $1 / 2^{-n / 2}=2^{n / 2}$ of such wavelengths and our grid of $N=2^{n}$ equidistant points resolves each such wavelength using $N / 2^{n / 2}=2^{n / 2}$ grid points. Therefore, the randomness of our quasirandom external potential of Eq. (6) as well as its FDM resolution grows exponentially with the number of qubits $n$. At the same time, all FDM errors (using an FDM representation of the Laplace operator, the trapezoidal rule for integration, and so on) decrease polynomially with the grid spacing $1 / N=2^{-n}$ and thus exponentially with $n$. Therefore, with growing values of $n$, our FDM representation of Eq. (1) converges exponentially fast to the continuous problem and the accuracy of randomness in Eq. (6) grows exponentially. Our proposal is to solve this problem for the strongly disordered regime $s_{1} / s_{2} \approx 1$ in Eq. (6), i.e., compute the corresponding ground states, using an increasing number $n=4,6,8, \ldots$ Based on the numerical analysis of Fig. 3, we conjecture that MPSs require resources $\mathcal{N}[$ MPS $] \propto \operatorname{poly}\left(\kappa_{1}\right)=\operatorname{poly}\left(2 \pi \times 2^{n / 2}\right)=\exp (n)$ growing exponentially with $n$, whereas the quantum ansatz of Fig. 1(b) just needs resources $\mathcal{N}[\mathrm{QA}] \propto \log \left(\kappa_{1}\right)=\log \left(2 \pi \times 2^{n / 2}\right)=$ $\operatorname{poly}(n)$ increasing polynomially with $n$. This calculation can be used to demonstrate quantum supremacy as, by successively increasing $n$, the limit of what is computationally possible on a classical computer (using MPSs or exact classi- cal methods) is reached quickly and our quantum ansatz on a quantum computer remains efficient far beyond this classical limit.

The quantum hardware requirements for this quantum supremacy calculation go beyond the current capabilities of available NISQ devices [73]. Nevertheless, the superior performance of our quantum ansatz is relevant for current NISQ devices as only the most efficient variational states can succeed in the presence of the current experimental errors. We test the feasibility of nonlinear VQC on NISQ devices by calculating the ground state of Eq. (1) for a simple harmonic potential and a single variational parameter on an IBM Q device utilizing further network optimizations (see [35] for details). The experimental implementation of the nonlinear VQC algorithm is able to identify the optimal variational parameter with an error of less than $10 \%$, leading to excellent agreement of the ground-state solutions with exact numerical solutions [cf. Figs. 1(c) and 1(d)].

The methods presented here are readily modified to twoand three-dimensional problems, with an overhead scaling linearly in the number of dimensions, and can be applied to a broad range of nonlinear terms and differential operators. An exciting prospect for future work would be to utilize intermediate-scale quantum computers to solve nonlinear problems on grid sizes beyond the scope of conventional computers.

M.L. and D.J. are grateful for funding from the Networked Quantum Information Technologies Hub of the UK National Quantum Technology Programme as well as from the EPSRC grant "Tensor network theory for strongly correlated quantum systems" (No. EP/K038311/1). We acknowledge support from the EPSRC National Quantum Technology Hub in Networked Quantum Information Technology (Grant No. EP/M013243/1). M.K. and D.J. acknowledge financial support from the National Research Foundation and the Ministry of Education, Singapore.
[1] S. Wiggins, Introduction to Applied Nonlinear Dynamical Systems and Chaos (Springer, New York, 2003).

[2] L. A. Aguirre and C. Letellier, Modeling nonlinear dynamics and chaos: A review, Math. Probl. Eng. 2009, 238960 (2009).

[3] S. H. Strogatz, Nonlinear Dynamics and Chaos (Westview, Boulder, 2015).

[4] P. Lynch, The Emergence of Numerical Weather Prediction (Cambridge University Press, Cambridge, 2006).

[5] T. T. Warner, Numerical Weather and Climate Prediction (Cambridge University Press, Cambridge, 2011).

[6] R. A. Pielke, Sr., Mesoscale Meteorological Modeling (Academic, Waltham, 2013).

[7] L. M. Milne-Thomson, Theoretical Aerodynamics (Dover, New York, 1973).

[8] J. H. Ferziger and M. Perić, Computational Methods for Fluid Dynamics (Springer, Berlin, 2002).

[9] H. K. Versteeg and W. Malalasekera, An Introduction to Computational Fluid Dynamics (Pearson Education, Harlow, 2007).
[10] S. Lloyd, Universal quantum simulators, Science 273, 1073 (1996)

[11] A. Aspuru-Guzik, A. D. Dutoi, P. J. Love, and M. Head-Gordon, Simulated quantum computation of molecular energies, Science 309, 1704 (2005).

[12] I. Kassal, S. P. Jordan, P. J. Love, M. Mohseni, and A. AspuruGuzik, Polynomial-time quantum algorithm for the simulation of chemical dynamics, Proc. Natl. Acad. Sci. USA 105, 18681 (2008)

[13] A. W. Harrow, A. Hassidim, and S. Lloyd, Quantum Algorithm for Linear Systems of Equations, Phys. Rev. Lett. 103, 150502 (2009).

[14] D. W. Berry, High-order quantum algorithm for solving linear differential equations, J. Phys. A: Math. Theor. 47, 105301 (2014).

[15] D. W. Berry, A. M. Childs, R. Cleve, R. Kothari, and R. D. Somma, Simulating Hamiltonian Dynamics with a Truncated Taylor Series, Phys. Rev. Lett. 114, 090502 (2015). 
[16] A. Montanaro and S. Pallister, Quantum algorithms and the finite element method, Phys. Rev. A 93, 032324 (2016).

[17] I. D. Kivlichan, N. Wiebe, R. Babbush, and A. Aspuru-Guzik, Bounding the costs of quantum simulation of many-body physics in real space, J. Phys. A: Math. Theor. 50, 305301 (2017).

[18] A. M. Childs, R. Kothari, and R. D. Somma, Quantum algorithm for systems of linear equations with exponentially improved dependence on precision, SIAM J. Comput. 46, 1920 (2017).

[19] A. Peruzzo, J. McClean, P. Shadbolt, M.-H. Yung, X.-Q. Zhou, P. J. Love, A. Aspuru-Guzik, and J. L. O'Brien, A variational eigenvalue solver on a photonic quantum processor, Nat. Commun. 5, 4213 (2014).

[20] J. R. McClean, J. Romero, R. Babbush, and A. Aspuru-Guzik, The theory of variational hybrid quantum-classical algorithms, New J. Phys. 18, 023023 (2016).

[21] P. J. J. O’Malley, R. Babbush, I. D. Kivlichan, J. Romero, J. R. McClean R. Barends, J. Kelly, P. Roushan, A. Tranter, N. Ding et al., Scalable Quantum Simulation of Molecular Energies, Phys. Rev. X 6, 031007 (2016).

[22] J. M. Kreula, S. R. Clark, and D. Jaksch, Non-linear quantumclassical scheme to simulate non-equilibrium strongly correlated fermionic many-body dynamics, Sci. Rep. 6, 32940 (2016).

[23] J. M. Kreula, L. García-Álvarez, L. Lamata, S. R. Clark, E. Solano, and D. Jaksch, Few-qubit quantum-classical simulation of strongly correlated lattice fermions, EPJ Quantum Technol. 3, 11 (2016).

[24] Y. Li and S. C. Benjamin, Efficient Variational Quantum Simulator Incorporating Active Error Minimization, Phys. Rev. X 7, 021050 (2017).

[25] A. Kandala, A. Mezzacapo, K. Temme, M. Takita, M. Brink, J. M. Chow, and J. M. Gambetta, Hardware-efficient variational quantum eigensolver for small molecules and quantum magnets, Nature (London) 549, 242 (2017).

[26] J. I. Colless, V. V. Ramasesh, D. Dahlen, M. S. Blok, M. E. Kimchi-Schwartz, J. R. McClean, J. Carter, W. A. de Jong, and I. Siddiqi, Computation of Molecular Spectra on a Quantum Processor with an Error-Resilient Algorithm, Phys. Rev. X 8, 011021 (2018).

[27] E. F. Dumitrescu, A. J. McCaskey, G. Hagen, G. R. Jansen, T. D. Morris, T. Papenbrock, R. C. Pooser, D. J. Dean, and P. Lougovski, Cloud Quantum Computing of an Atomic Nucleus, Phys. Rev. Lett. 120, 210501 (2018).

[28] C. Hempel, C. Maier, J. Romero, J. McClean, T. Monz, H. Shen, P. Jurcevic, B. P. Lanyon, P. Love, R. Babbush, A. Aspuru-Guzik, R. Blatt, and C. F. Roos, Quantum Chemistry Calculations on a Trapped-Ion Quantum Simulator, Phys. Rev. X 8, 031022 (2018).

[29] J. Romero, R. Babbush, J. R. McClean, C. Hempel, P. J. Love, and A. Aspuru-Guzik, Strategies for quantum computing molecular energies using the unitary coupled cluster ansatz, Quantum Sci. Technol. 4, 014008 (2018).

[30] S. McArdle, T. Jones, S. Endo, Y. Li, S. Benjamin, and X. Yuan, Variational ansatz-based quantum simulation of imaginary time evolution, npj Quantum Inf. 5, 75 (2019).

[31] S. Endo, Y. Li, S. Benjamin, and X. Yuan, Variational quantum simulation of general processes, arXiv:1812.08778.
[32] M.-C. Chen, M. Gong, X.-S. Xu, X. Yuan, J.-W. Wang, C. Wang, C. Ying, J. Lin, Y. Xu, Y. Wu, S. Wang, H. Deng, F. Liang, C.-Z. Peng, S. C. Benjamin, X. Zhu, C.-Y. Lu, and J.-W. Pan, Demonstration of adiabatic variational quantum computing with a superconducting quantum coprocessor, arXiv:1905.03150.

[33] A. Georges, G. Kotliar, W. Krauth, and M. J. Rozenberg, Dynamical mean-field theory of strongly correlated fermion systems and the limit of infinite dimensions, Rev. Mod. Phys. 68, 13 (1996).

[34] G. Kotliar, S. Y. Savrasov, K. Haule, V. S. Oudovenko, O. Parcollet, and C. A. Marianetti, Electronic structure calculations with dynamical mean-field theory, Rev. Mod. Phys. 78, 865 (2006).

[35] See Supplemental Material at http://link.aps.org/supplemental/ 10.1103/PhysRevA.101.010301 for details about the IBM quantum computations, adder circuit for the kinetic energy, nonlinear VQC for the Burgers equation, MPS ansatz, sampling error, fidelity $\mathcal{F}$ optimization, and number of variational parameters.

[36] I. Griva, S. G. Nash, and A. Sofer, Linear and Nonlinear Optimization (SIAM, Philadelphia, 2009).

[37] E. P. Gross, Structure of a quantized vortex in boson systems, Nuovo Cimento 20, 454 (1961).

[38] L. P. Pitaevskii, Vortex lines in an imperfect Bose gas, Sov. Phys. JETP 13, 451 (1961).

[39] A. J. Leggett, Bose-Einstein condensation in the alkali gases: Some fundamental concepts, Rev. Mod. Phys. 73, 307 (2001).

[40] L. Pitaevskii and S. Stringari, Bose-Einstein Condensation (Clarendon, Oxford, 2003).

[41] A. Scott, Encyclopedia of Nonlinear Science (Routledge, New York, 2005)

[42] G. Agrawal, Nonlinear Fiber Optics (Academic, Waltham, 2013).

[43] K. Viebahn, M. Sbroscia, E. Carter, J.-C. Yu, and U. Schneider, Matter-Wave Diffraction from a Quasicrystalline Optical Lattice, Phys. Rev. Lett. 122, 110404 (2019).

[44] W. H. Press, S. A. Teukolsky, W. T. Vetterling, and B. P. Flannery, Numerical Recipes in C (Cambridge University Press, New York, 1992).

[45] C. Grossmann, H.-G. Roos, and M. Stynes, Numerical Treatment of Partial Differential Equations (Springer, Berlin, 2007).

[46] A. Iserles, A First Course in the Numerical Analysis of Differential Equations (Cambridge University Press, Cambridge, 2009).

[47] F. Verstraete, V. Murg, and J. I. Cirac, Matrix product states, projected entangled pair states, and variational renormalization group methods for quantum spin systems, Adv. Phys. 57, 143 (2008).

[48] I. V. Oseledets, Approximation of $2^{d} \times 2^{d}$ matrices using tensor decomposition, SIAM J. Matrix Anal. Appl. 31, 2130 (2010).

[49] I. V. Oseledets, Tensor-train decomposition, SIAM J. Sci. Comput. 33, 2295 (2011).

[50] R. Orús, A practical introduction to tensor networks: Matrix product states and projected entangled pair states, Ann. Phys. (NY) 349, 117 (2014).

[51] B. N. Khoromskij, $O(d \log N)$-quantics approximation of $N-d$ tensors in high-dimensional numerical modeling, Constr. Approx. 34, 257 (2011). 
[52] I. V. Oseledets, Constructive representation of functions in lowrank tensor formats, Constr. Approx. 37, 1 (2013).

[53] A. K. Ekert, C. M. Alves, D. K. L. Oi, M. Horodecki, P. Horodecki, and L. C. Kwek, Direct Estimations of Linear and Nonlinear Functionals of a Quantum State, Phys. Rev. Lett. 88, 217901 (2002).

[54] C. M. Alves, P. Horodecki, D. K. L. Oi, L. C. Kwek, and A. K. Ekert, Direct estimation of functionals of density operators by local operations and classical communication, Phys. Rev. A 68 , 032306 (2003).

[55] J. M. Spall, Multivariate stochastic approximation using a simultaneous perturbation gradient approximation, IEEE Trans. Automat. Contr. 37, 332 (1992).

[56] M. Lubasch, P. Moinier, and D. Jaksch, Multigrid renormalization, J. Comput. Phys. 372, 587 (2018).

[57] T. E. O'Brien, B. Senjean, R. Sagastizabal, X. Bonet-Monroig, A. Dutkiewicz, F. Buda, L. DiCarlo, and L. Visscher, Calculating energy derivatives for quantum chemistry on a quantum computer, arXiv:1905.03742.

[58] K. Mitarai, Y. O. Nakagawa, and W. Mizukami, Theory of analytical energy derivatives for the variational quantum eigensolver, arXiv:1905.04054.

[59] C. Schön, E. Solano, F. Verstraete, J. I. Cirac, and M. M. Wolf, Sequential Generation of Entangled Multiqubit States, Phys. Rev. Lett. 95, 110503 (2005).

[60] C. Schön, K. Hammerer, M. M. Wolf, J. I. Cirac, and E. Solano, Sequential generation of matrix-product states in cavity QED, Phys. Rev. A 75, 032311 (2007).

[61] M. C. Bañuls, D. Pérez-García, M. M. Wolf, F. Verstraete, and J. I. Cirac, Sequentially generated states for the study of twodimensional systems, Phys. Rev. A 77, 052306 (2008).

[62] M. Reck, A. Zeilinger, H. J. Bernstein, and P. Bertani, Experimental Realization of Any Discrete Unitary Operator, Phys. Rev. Lett. 73, 58 (1994).

[63] A. Barenco, C. H. Bennett, R. Cleve, D. P. DiVincenzo, N.
Margolus, P. Shor, T. Sleator, J. A. Smolin, and H. Weinfurter, Elementary gates for quantum computation, Phys. Rev. A 52, 3457 (1995).

[64] V. V. Shende, S. S. Bullock, and I. L. Markov, Synthesis of quantum-logic circuits, IEEE Trans. Comput.-Aid. Design 25, 1000 (2006).

[65] R. Iten, R. Colbeck, I. Kukuljan, J. Home, and M. Christandl, Quantum circuits for isometries, Phys. Rev. A 93, 032318 (2016).

[66] B. Deissler, M. Zaccanti, G. Roati, C. D’Errico, M. Fattori, M. Modugno, G. Modugno, and M. Inguscio, Delocalization of a disordered bosonic system by repulsive interactions, Nat. Phys. 6, 354 (2010).

[67] A. J. Daley, C. Kollath, U. Schollwöck, and G. Vidal, Time-dependent density-matrix renormalization-group using adaptive effective Hilbert spaces, J. Stat. Mech. (2004) P04005.

[68] S. Trotzky, Y.-A. Chen, A. Flesch, I. P. McCulloch, U. Schollwöck, J. Eisert, and I. Bloch, Probing the relaxation towards equilibrium in an isolated strongly correlated onedimensional Bose gas, Nat. Phys. 8, 325 (2012).

[69] J. Schachenmayer, B. P. Lanyon, C. F. Roos, and A. J. Daley, Entanglement Growth in Quench Dynamics with Variable Range Interactions, Phys. Rev. X 3, 031015 (2013).

[70] M. A Nielsen and I. L. Chuang, Quantum Computation and Quantum Information (Cambridge University Press, Cambridge, 2010).

[71] B. Kramer and A. MacKinnon, Localization: Theory and experiment, Rep. Prog. Phys. 56, 1469 (1993).

[72] G. Vidal, Efficient Classical Simulation of Slightly Entangled Quantum Computations, Phys. Rev. Lett. 91, 147902 (2003).

[73] A. W. Cross, L. S. Bishop, S. Sheldon, P. D. Nation, and J. M. Gambetta, Validating quantum computers using randomized model circuits, Phys. Rev. A 100, 032328 (2019). 